Topics in Applied Physics Volume 70

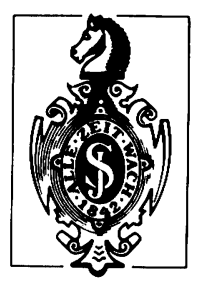


Volume 57 Strong and Ultrastrong Magnetic Fields and Their Applications Editor: F. Herlach

Volume 58 Hot-Electron Transport in Semiconductors Editor: L. Reggiani

Volume 59 Tunable Lasers

Editors: L.F. Mollenauer and J. C. White

Volume 60 Ultrashort Laser Pulses and Applications Editor: W. Kaiser

Volume 61 Photorefractive Materials and Their Applications I Fundamental Phenomena

Editors: P. Günter and J.-P. Huignard

Volume 62 Photorefractive Materials and Their Applications II Survey of Applications

Editors: P. Günter and J.-P. Huignard

Volume 63 Hydrogen in Intermetallic Compounds I

Electronic, Thermodynamic and Crystallographic

Properties, Preparation

Editor: L. Schlapbach

Volume 64 Sputtering by Particle Bombardment III

Characteristics of Sputtered Particles, Technical Applications

Editors: R. Behrisch and K. Wittmaack

Volume 65 Laser Spectroscopy of Solids II

Editor: W. M. Yen

Volume 66 Light Scattering in Solids V

Superlattices and Other Microstructures

Editors: M. Cardona and G. Güntherodt

Volume 67 Hydrogen in Intermetallic Compounds II

Surface and Dynamic Properties, Applications

Editor: L. Schlapbach

Volume 68 Light Scattering in Solids VI

Recent Results, Including High- $\mathrm{T}_{\mathrm{c}}$ Superconductivity

Editors: M. Cardona and G. Güntherodt

Volume 69 Unoccupied Electronic States

Editors: J. C. Fuggle and J. E. Inglesfield

Volume 70 Dye Lasers: 25 Years

Editor: M. Stuke

Volume 71 The Monte Carlo Method in Condensed Matter Physics

Editor: K. Binder

Volumes 1-56 are listed on the back inside cover 


\section{Dye Lasers: 25 Years}

Edited by Michael Stuke

With 151 Figures

Springer-Verlag

Berlin Heidelberg New York London Paris

Tokyo Hong Kong Barcelona Budapest 


\section{Contents}

1. Femtosecond Broadband Absorption Spectroscopy of Photodissociating Molecules

By J. Glownia, J. Misewich, R. Walkup, M. Kaschke and

P. Sorokin (With 12 Figures) .............. 1

1.1 Probing the Dissociation Process: TlI Transient Spectra . . . 3

1.2 "Impact" and "Statistical" Transient Absorption Spectra. . . 6

1.3 Extracting the Difference Potential. . . . . . . . . . . . 8

1.4 Examples of "Statistical" Spectra: $\mathrm{Bi}_{2} \ldots \ldots \ldots 11$

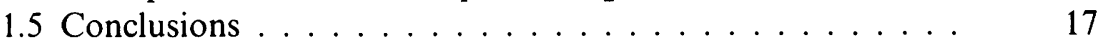

References....................... 17

2. Dye Lasers and Laser Dyes in Physical Chemistry

By F.P. Schäfer (With 22 Figures). . . . . . . . . . . . . . . . . 19

2.1 The Wavelength Limits of Dye Lasers. . . . . . . . . . . . . . 19

2.2 Limitations by Radiationless Transitions . . . . . . . . . . . . . . 23

2.3 The Smallest and Largest Dye Lasers . . . . . . . . . . . . . . . . 26

2.4 Increasing the Efficiency of Dye Lasers . . . . . . . . . . . . 30

2.5 Photodegradation of Laser Dyes . . . . . . . . . . . . . . . 33

References.................... 36

3. Single-Atom Experiments and the Test

of Fundamental Quantum Physics

By H. Walther (With 10 Figures) . . . . . . . . . . . . . . 37

3.1 Overview . . . . . . . . . . . . . . . . . 37

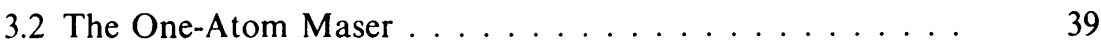

3.3 Dynamics of a Single Atom . . . . . . . . . . . . . . . . . . . 41

3.4 A New Source of Nonclassical Light . . . . . . . . . . . . . 42

3.5 Experimental Results - A Nonclassical Beam of Atoms . . . 44

3.6 A New Probe of Complementarity in Quantum Mechanics . $\quad 50$

3.7 Summary and Conclusion . . . . . . . . . . . . . . . 54

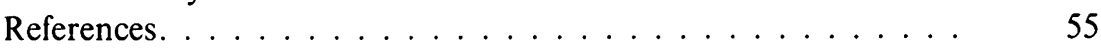

4. Regioselective Photochemistry in Weakly Bonded Complexes

By S. K. Shin, Y. Chen, E. Böhmer and C. Wittig

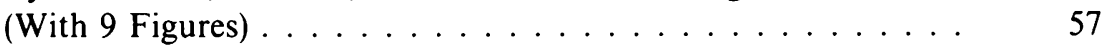

4.1 Introduction ........................ 57 
4.2 End-On $\mathrm{CO}_{2}-\mathrm{HCl}$ and Broadside $\mathrm{CO}_{2}-\mathrm{HBr}$. . . . . . . . 61

4.2.1 Product State Distributions .............................. 64

4.2.2 Time-Resolved Product Buildup. . . . . . . . . . . . . 65

4.2.3 Theoretical Considerations . . . . . . . . . . . . . . 65

$4.3 \mathrm{~N}_{2} \mathrm{O}-\mathrm{HI}: \mathrm{OH}+\mathrm{N}_{2}$ vs. $\mathrm{NH}+\mathrm{NO} \ldots \ldots . \ldots 68$

4.3.1 Product State Distributions ............... 69

4.3.2 The Product Branching Ratio: $\mathrm{OH}+\mathrm{N}_{2}$ vs. $\mathrm{NH}+\mathrm{NO} 71$

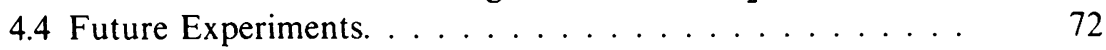

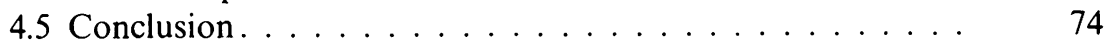

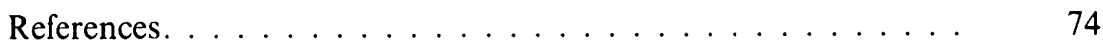

5. Novel Resonator Design for Femtosecond Lasers

By V. Magni, S. De Silvestri, A. Cybo-Ottone, M. Nisoli and O. Svelto (With 6 Figures) . . . . . . . . . . . . . . . . . . . . . 77

5.1 Introduction . . . . . . . . . . . . . . . . . . . . . 77

5.2 One-Folding Resonators. . . . . . . . . . . . . . . . . . 78

5.3 Two-Folding Resonators. . . . . . . . . . . . . . . . . . 82

5.4 Conclusions . . . . . . . . . . . . . . . . . . . . . . . . . 85

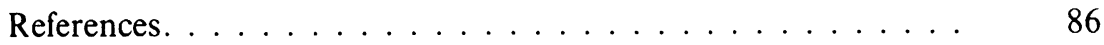

6. Distortion of Femtosecond Pulse Fronts in Lenses

By Z. Bor and Z.L. Horváth (With 4 Figures) . . . . . . . . . . . 87

6.1 Diffraction of a Femtosecond Pulse by a Dispersive Lens . . 87

6.2 Results of the Calculations . . . . . . . . . . . . . . . . . . . 89

6.2.1 Distortion of the Pulse Front ........... 89

6.2.2 Boundary Wave Pulse on the Optical Axis . . . . . . . 90

6.2 .3 Pulse Shape in the Focus. . . . . . . . . . . . . . . 91

6.2.4 Intensity Distribution in the Focal Plane . . . . . . . 91

6.2.5 Intensity Distribution in a Plane Neighboring the Focal Plane . . . . . . . . . . . . . . . 92

6.3 Conclusions . . . . . . . . . . . . . . . . . . . . . . . . . . 93

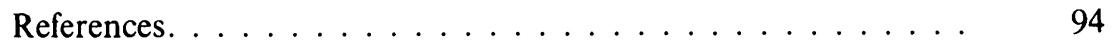

7. Infrared Dye Lasers for the Wavelength Range 1-2 $\mu \mathrm{m}$

By T. Elsaesser and W. Kaiser (With 8 Figures) . . . . . . . . . . . 95

7.1 Photophysics of Infrared Dyes . . . . . . . . . . . . . . . . . . 95

7.2 Infrared Dye Lasers . . . . . . . . . . . . . . . . . . . . . . . . . 98

7.2.1 Nanosecond Infrared Dye Lasers . . . . . . . . . . . 99

7.2.2 Synchronously Pumped Picosecond and Subpicosecond Systems . . . . . . . . . . . 99

7.2.3 Picosecond Dye Lasers Pumped in a Traveling-Wave Geometry . . . . . . . . . . 101

7.2.4 Nonlinear Frequency Conversion with Infrared Dye Lasers . . . . . . . . . . . . . . . . 106

References. . . . . . . . . . . . . . . . . 108 
8. Propagation of Femtosecond Light Pulses Through Dye Amplifiers

By B. Wilhelmi (With 13 Figures). . . . . . . . . . . 111

8.1 Introduction . . . . . . . . . . . . . . . . 111

8.2 Interaction in the Rate-Equation Approximation . . . . . . 116

$\begin{array}{ll}\text { 8.3 Interaction of Very Short Light Pulses with Dye Solutions . } & 117\end{array}$

8.4 Chirped and Stretched Pulse Amplification. . . . . . . . . 121

8.5 Conclusions . . . . . . . . . . . . . . . . . . . . . . . 125

References. . . . . . . . . . . . . . . . . 126

9. Terawatt-Class Hybrid Dye/Excimer Lasers

By S. Szatmári (With 5 Figures). . . . . . . . . . . . . . . . . . . 129

9.1 Input Pulse Generation by Pulsed Lasers. . . . . . . . . . . . 130

9.2 Short-Pulse Amplification Properties of Excimers . . . . . . . 132

9.3 Critical Issues for a TW Excimer Amplifier. . . . . . . . . . . . . 133

9.4 Spatially Evolving Chirped-Pulse Amplification . . . . . . . . 134

9.5 Off-Axis Amplification and Phase-Locked Multiplexing . . . 136

References. ..................... 140

10. Blue-Green Dye Laser Seeded Operation

of a Terawatt Excimer Amplifier

By F.K. Tittel, T. Hofmann, T.E. Sharp, P.J. Wisoff, W.L. Wilson and G. Szabó (With 5 Figures) . . . . . . . . . . . . . . . . 141

10.1 Introduction . . . . . . . . . . . . . . . . . . . . . . 141

10.2 Dye Laser System . . . . . . . . . . . . . . . . . . . . . . . . 142

10.2.1 Oscillator Section . . . . . . . . . . . . . 142

10.2.2 High-Performance Subpicosecond Dye Amplifier

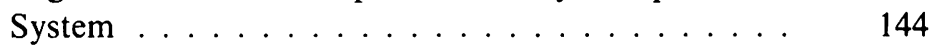

$10.3 \mathrm{XeF}(\mathrm{C}-\mathrm{A})$ Experiments . . . . . . . . . . . . . . . 146

10.3.1 Experimental Arrangement . . . . . . . . . . . . 146

10.3.2 Gain Measurements. . . . . . . . . . . . . . . . . . . 146

10.3 .3 Energy Extraction. . . . . . . . . . . . . . . . . . 148

10.4 Conclusions. . . . . . . . . . . . . . . . . . . . . . . 150

References. ..................... 151

\section{Atomic Optics with Tunable Dye Lasers}

By V.S. Letokhov (With 9 Figures) . . . . . . . . . . . . . . . . . . . . 153

11.1 Introduction and History. . . . . . . . . . . . . 153

11.2 Radiation Force Acting on Atoms in a Resonant Light Field 155

a) Plane Wave . . . . . . . . . . . . . . . . . . . . 155

b) Gaussian Laser Beam . . . . . . . . . . . . . . . . . . . . . . 156

c) Plane Standing Light Wave. . . . . . . . . . . . . . . . 157

11.3 Collimation. . . . . . . . . . . . . . . 157

11.4 Focusing an Atomic Beam; Imaging an Atomic Source. . . 159

11.5 Atomic Beam Reflection ................ 162 
11.6 Conclusion and Prospects $\ldots \ldots \ldots \ldots \ldots \ldots$

References.

12. Laser Spectroscopy of Small Molecules

By V. Beutel, G. Bhale, W. Demtröder, H.-A. Eckel, J. Gress and M. Kuhn (With 10 Figures) . . . . . . . . . . . . .

12.1 Introduction . . . . . . . . . . . . . . . . . . . . 169

12.2 Determination of Singlet-Triplet Potentials of Alkali Molecules . . . . . . . . . . . . . . . . . . 170

12.3 Isotope-Selective Spectroscopy of $\mathrm{Ag}_{2}$ Dimers . . . . . . . 172

12.4 Sub-Doppler Spectroscopy of the Sodium Cluster . . . . . 176

References. . . . . . . . . . . . . . . . . . . . . . 180

13. In Situ Gas-Phase Diagnostics

by Coherent Anti-Stokes Raman Scattering

By W. Richter (With 9 Figures) . . . . . . . . . . . . . . . 183

13.1 Introduction . . . . . . . . . . . . . . . . 183

13.2 Coherent Anti-Stokes Raman Scattering. . . . . . . . . . . 184

13.3 Experimental Considerations . . . . . . . . . . . . 185

13.4 Experimental Results . . . . . . . . . . . . . . . . . . . 189

13.5 Summary and Conclusions. . . . . . . . . . . . . . . . 192

References. . . . . . . . . . . . . . . . . . . . . 194

14. High-Resolution Spectroscopy at Short Wavelengths

Using Pulsed Dye Lasers

By S. Svanberg (With 5 Figures) _. . . . . . . . . . . . . 195

14.1 Generation of UV/VUV Radiation . . . . . . . . . . . . 196

14.1.1 Sum-Frequency Generation in Crystals . . . . . . . 196

14.1.2 Frequency Conversion in Gases. . . . . . . . . . . . 196

14.2 High-Resolution Laser Spectroscopy Using Pulsed Dye

Lasers . . . . . . . . . . . . . . . . . . . . . . . . . . . . 197

14.2.1 Optical Double Resonance Experiments. . . . . . . . 197

14.2.2 Level-Crossing Measurements. . . . . . . . . . . . . 197

14.2.3 Quantum-Beat Measurements. . . . . . . . . . . . . 199

14.2.4 Time-Resolved Studies on Atoms

Formed by Short-Wavelength Dissociation . . . . . 200

14.3 Conclusion . . . . . . . . . . . . . . . . . . 201

References. . . . . . . . . . . . . . . . . . . 202

15. Doppler-Free Spectroscopy of Large Polyatomic Molecules and van der Waals Complexes

By H.J. Neusser, E. Riedle, T. Weber and E.W. Schlag

(With 5 Figures) . . . . . . . . . . . . . . . . . . 205

15.1 Introduction . . . . . . . . . . . . . . . . 205

15.2 Doppler-Free Two-Photon Spectroscopy of Benzene . . . 205 
15.3 Doppler-Free UV Spectroscopy of Benzene-Noble-Gas van der Waals Clusters . . . . . . . . . . . . . . . . . 209

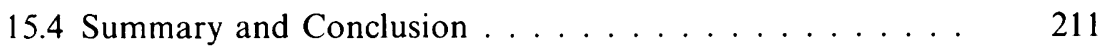

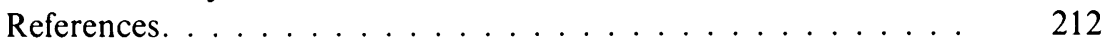

16. Electro-optic and Photoconductive Sampling of Ultrafast Photodiodes with Femtosecond Laser Pulses

By J. Kuhl, M. Klingenstein, M. Lambsdorff, J. Rosenzweig,

C. Moglestue and A. Axmann (With 8 Figures). . . . . . . . 213

16.1 Introduction . . . . . . . . . . . . . . . . . . . . 213

16.2 Photoconductive Switches ............... 213

16.3 Electro-optical Sampling . . . . . . . . . . . . . 217

16.4 Characterization of MSM Photodiodes .......... 218

16.4.1 MSM Schottky Photodetectors........... 218

16.4.2 Monte Carlo Simulation of the Transport. . . . . . 219

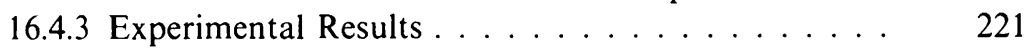

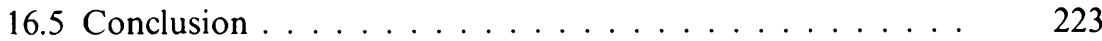

References. ....................... 223

17. Ultrafast Spectroscopy of Plasmas Generated by Very Intense

Femtosecond Dye Laser Pulses

By D. von der Linde (With 6 Figures) . . . . . . . . . . . . . . . 225

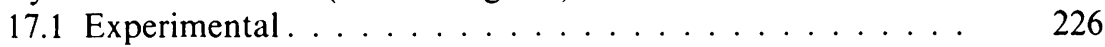

17.2 Characteristics of the Reflected Light . . . . . . . . . . . . 228

17.3 Collinear Pump-Probe Experiments ........... 230

17.4 Non-collinear Pump-Probe Experiments ......... 231

17.5 Conclusions. . . . . . . . . . . . . . . . . . . . . . 234

References. . . . . . . . . . . . . . . . . . 234

18. Dye Laser Spectroscopy of Isolated Atoms and Ions in Liquid Helium

By G. zu Putlitz and M. R. Beau (With 5 Figures). . . . . . . . . 237

18.1 Effects of Pressure on Spectral Lines. . . . . . . . . . . . . 237

18.2 Impurities in Liquid Helium. . . . . . . . . . . . . . . . 239

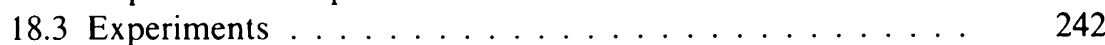

18.4 Spectroscopic Results. . . . . . . . . . . . . . . . . . 244

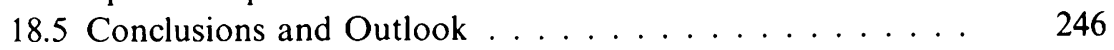

References. ...................... 246 


\title{
15. Doppler-Free Spectroscopy of Large Polyatomic Molecules and van der Waals Complexes
}

\author{
H.J. Neusser, E. Riedle, T. Weber and E.W. Schlag \\ With 5 Figures
}

It is shown that high-resolution spectroscopy with tunable dye lasers leads to rotationally resolved electronic spectra of large molecular systems. Two-photon absorption of narrow-band cw light in an external cavity eliminates Doppler broadening in the $S_{1} \leftarrow S_{0}$ transition of the prototype organic molecule benzene. Several thousands of rotational lines in the room temperature spectrum are analyzed, providing spectroscopic constants with a hitherto inaccessible precision. Investigations of the homogeneous linewidth of individual rotational transitions reveals that Coriolis coupling plays an important role in the intramolecular energy redistribution process in this molecule. Aided by the reduced Doppler broadening in a skimmed cooled supersonic beam, rotationally resolved UV spectra of benzene-noble-gas van der Waals clusters were measured. These measurements yield precise information on the van der Waals bond lengths and structures of these complexes.

\subsection{Introduction}

The advent of narrow-band tunable dye lasers $[15.1,2]$ has caused a breakthrough in the precision of atomic spectroscopy and, more recently, has allowed high-resolution molecular spectroscopy of systems with many vibrational degrees of freedom to be performed. Several techniques have been developed that push gas-phase spectral resolution below the natural barrier set by Doppler broadening [15.3]. Spectroscopy in collimated beams, saturation spectroscopy, polarization spectroscopy and Doppler-free two-photon spectroscopy were originally demonstrated for atoms to reveal the underlying line structure of a Doppler-broadened transition. In this contribution we would like to summarize some of our important new results achieved for isolated organic molecules. Two different types of experiments are described leading to Doppler-free spectra of either a large polyatomic molecule at room temperature or of van der Waals complexes at low temperature. Typical examples for the spectra and the new information revealed are presented.

\subsection{Doppler-Free Two-Photon Spectroscopy of Benzene}

Following the successful application of Doppler-free methods in atomic physics, these techniques have rapidly gained importance in the spectroscopy of poly-

Topics in Applied Physics, Vol. 70

Dye Lasers: 25 Years Ed.: Dr. Michael Stuke

(C) Springer-Verlag Berlin Heidelberg 1992 
atomic molecules. Of the various methods mentioned above, Doppler-free twophoton absorption was the technique first successfully applied to large molecules [15.4]. One advantage of two-photon excitation is that narrow-band visible laser light can be used to observe electronic transitions occurring at UV photon energies. Indeed most polyatomic molecules, and in particular organic systems, begin to absorb in the UV part of the spectrum. Other important advantages are that all molecules within the interaction volume contribute to the Doppler-free signal independent of their velocity and that the Dopplerbroadened background (due to the absorption of two photons from one laser beam) can often be suppressed by proper choice of laser polarization $[15.5,6]$.

The typical experimental set-up for a Doppler-free two-photon experiment consists of a tunable laser light source with a frequency width narrower than the Doppler width (typically a frequency stabilized single-mode $\mathrm{cw}$ dye laser) and a gas cell that contains the low-pressure molecular gas under investigation. Elimination of the Doppler broadening is achieved by the absorption of two photons propagating in opposite directions. This is accomplished by placing the gas cell within a standing light field, e.g., in an external resonator as originally suggested by Vasilenko et al. [15.7] or by back reflection of the laser beam onto itself. For molecular spectroscopy, the sensitivity of the external resonator arrangement is an important advantage. The oscillator strength of a molecular electronic transition is distributed over many vibrational transitions according to the Franck-Condon principle. Furthermore, even at room temperature, tens of thousands of rovibrational ground state levels are populated and the effective population density of a single rovibronic state is smaller by four orders of magnitude than the total gas density. Finally, in many polyatomic molecules the fluorescence quantum efficiency is smaller than unity due to fast nonradiative processes occurring within the isolated molecule. The greater sensitivity of the external resonator with its light intensity enhancement of one order of magnitude is often the only way to raise the two-photon signal above the detection threshold in a cw experiment.

The scheme of a Doppler-free two-photon experiment with external cavity as used in our laboratory [15.8] is shown in Fig. 15.1. The external cavity is locked to the $\mathrm{cw}$ dye laser frequency with a technique developed by Hänsch and Couillaud [15.9]. Two-photon absorption is monitored by detection of UV fluorescence from the excited level. At room temperature the Doppler-free spectrum of a polyatomic molecule consists of many thousands of rotational lines which arise from the large number of thermally populated ground state levels. For demonstration a small portion $(10 \%)$ of the $14_{0}^{1}$ vibronic band of benzene with a resolution of $10 \mathrm{MHz}$ is shown in Fig. 15.2. Every line represents a single rotational transition. Typically the line density in the spectrum is more than 150 lines $/ \mathrm{cm}^{-1}$. In benzene, on an average, about 8 lines are located within the Doppler width of $1.6 \mathrm{GHz}$. For this reason every laser scan yields a tremendous number of data points which have to be rapidly transferred to and stored in a computer with sufficient memory. The complete spectrum is assembled by many individual laser scans of typically $30 \mathrm{GHz}\left(1 \mathrm{~cm}^{-1}\right)$. 


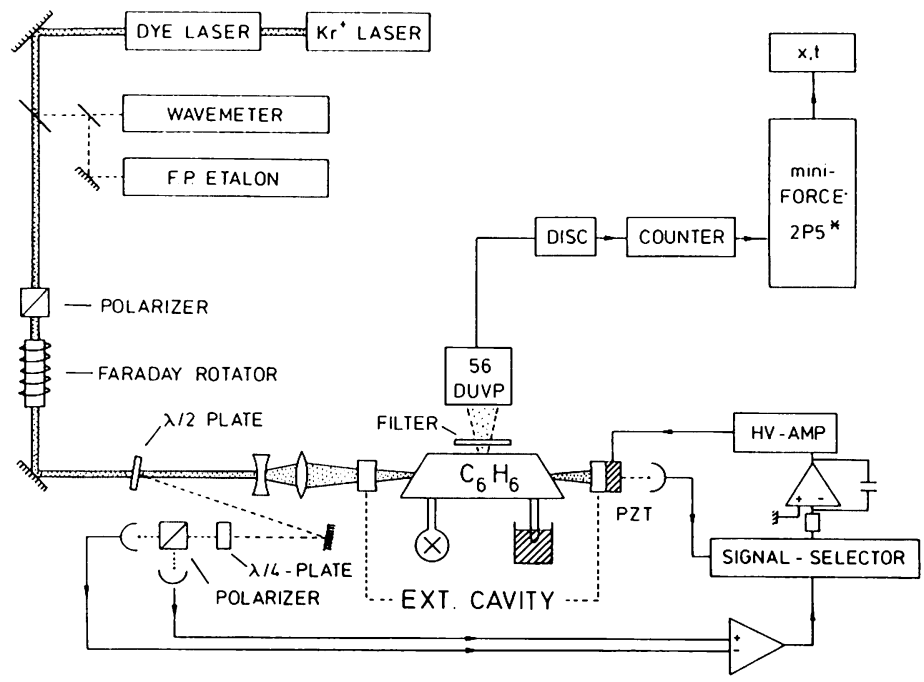

Fig. 15.1. Scheme of the experimental set-up for Doppler-free two-photon fluorescence excitation spectroscopy of polyatomic molecules. The cell containing the molecular gas is placed in an external concentric cavity, which creates the standing wave field for the two-photon absorption from counterpropagating light beams

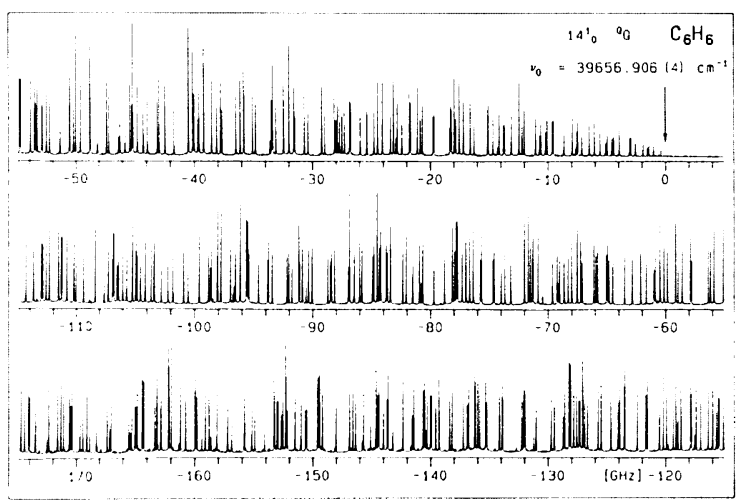

Fig. 15.2. Doppler-free twophoton spectrum of a small portion of the $14_{0}^{1}$ vibronic band in benzene, $\mathrm{C}_{6} \mathrm{H}_{6}$. Every line corresponds to an individual rotational transition and has been assigned

The rotational line spectrum can be described by a semirigid symmetric top Hamiltonian including quartic centrifugal distortion constants. A fit of the theoretical spectrum to the accurate line positions yields precise rotational constants and centrifugal distortion constants of the ground and excited electronic state. The precision is higher by two orders of magnitude than the constants obtained from conventional high-resolution but Doppler-limited UV spectroscopy [15.10]. A typical result for the rotational constants in the electronically excited $14^{1}$ state is $B_{14}^{\prime}=0.181284 \mathrm{~cm}^{-1}$ and $C_{14}^{\prime}$ $=0.090711 \mathrm{~cm}^{-1}$ with an accuracy of $10^{-6} \mathrm{~cm}^{-1}[15.11]$. 
In addition to the precise spectroscopic studies of molecular structure, Doppler-free two-photon absorption provides important information on molecular dynamics. After the inhomogeneous Doppler broadening is eliminated, the homogeneous linewidths of molecular transitions can be observed. At gas pressures of several mbar, the homogeneous linewidth is governed by collisional broadening, but at lower gas densities the linewidth is determined by dynamic processes taking place within the isolated molecule. In recent years there has been a growing interest in the study of intramolecular dynamics within the dense bound level structure typical of large molecules. Dynamics in a molecule with discrete level structure is of particular interest in photochemistry in order to understand the photophysical primary processes which occur before a polyatomic molecule undergoes a chemical reaction. Nonradiative processes such as internal conversion (IC), intersystem crossing (ISC) and intramolecular vibrational energy redistribution (IVR) are internal energy redistribution processes important for every chemist.

It is the goal of high-resolution spectroscopy to elucidate the abovementioned dynamic processes. The precise investigation of these processes is only possible if the excitation is selective enough to lead to an excitation of single defined quantum states.

In Fig. 15.3 the measured homogeneous linewidths of three individual rotational transitions in the $14^{1} 1^{2}$ vibrational state at a high excess energy of

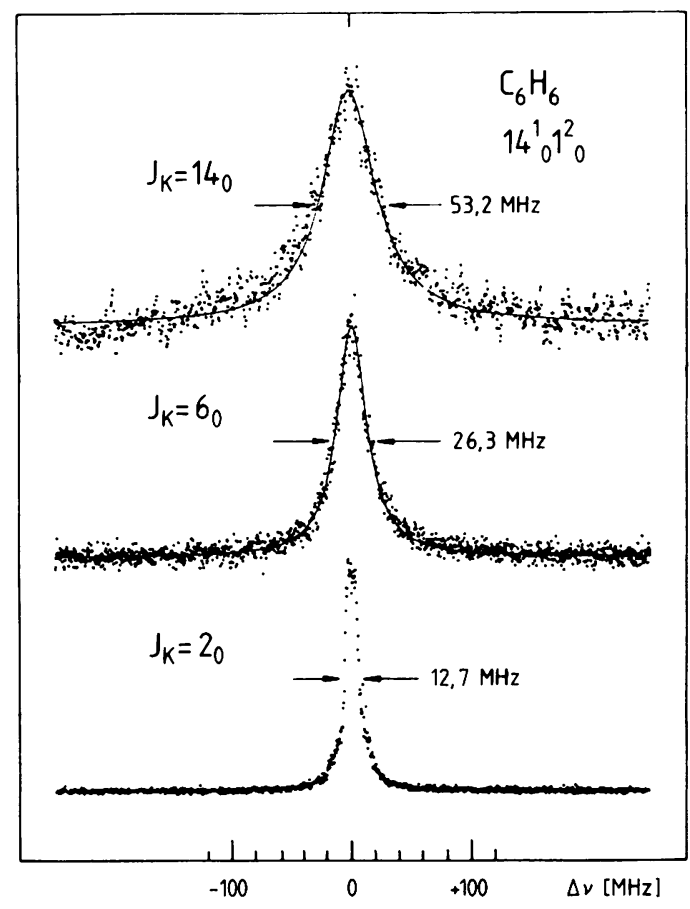

Fig. 15.3. Measured homogeneous linewidth of three rotational lines of the $14_{0}^{1} 1_{0}^{2}$ band at an excess energy of $3412 \mathrm{~cm}^{-1}$. The solid lines represent a Lorentzian curve fitted to the experimental points. The linewidth increases with increasing $J$ due to Coriolis coupling [15.8] 
$3412 \mathrm{~cm}^{-1}$ in the electronically excited state of benzene are shown [15.8]. The transitions lead to the defined states with rotational quantum numbers $J^{\prime}{ }^{\prime}$ ' $=2_{0}, 6_{0}$, and $14_{0}$, respectively. It is clearly recognized that the Lorentzian shape (solid line) of the lines increases from 12.7 to $53.2 \mathrm{MHz}$ with increasing $J^{\prime}$ quantum number. This situation resembles the rotational predissociation phenomenon typical for small molecules [15.12]. Here, in the large molecule benzene the rotational dependence of the linewidth clearly points to parallel Coriolis coupling. Thus, it is seen that Coriolis coupling, i.e. coupling of vibrational states through the rotation of the molecules, is an important coupling mechanism leading to intramolecular redistribution processes (IVR) of energy in large molecules.

\subsection{Doppler-Free UV Spectroscopy of Benzene-Noble-gas van der Waals Clusters}

In the past decade there has been growing interest in the spectroscopy of van der Waals complexes. The production of isolated atomic and molecular clusters has become feasible in cooled supersonic jet expansions [15.13]. The cold molecular beam provides another important advantage for high-resolution spectroscopy, i.e. the reduction of the transversal velocity distribution and consequently of Doppler broadening by selecting the central part of the beam with a skimmer. Thus, elimination of Doppler broadening can be obtained without using nonlinear techniques such as saturation spectroscopy or Doppler-free twophoton absorption.

In a supersonic jet expansion it is not possible to produce a single-cluster species, but rather, in addition to the monomer constituents of the gas mixture, a variety of clusters of differing composition and size is produced. The spectra of the different species may overlap and it is often difficult to distinguish them in the fluorescence excitation spectra. For this reason we combined high-resolution spectroscopy with resonance-enhanced two-photon ionization [15.14]. The produced ions can be mass-analyzed and integrated for a selected mass. This leads to highly resolved $(\Delta v=130 \mathrm{MHz})$ mass-selected two-photon ionization spectra reflecting the UV intermediate state spectrum of the selected cluster species.

Pulsed excitation with high spectral resolution is necessary for an efficient two-photon ionization. Light having these properties is provided by amplification of the single-mode $\mathrm{cw}$ laser in a three-stage amplifier system pumped by an excimer laser. The light pulses are Fourier-transform-limited with a bandwidth of $80 \mathrm{MHz}$ and a peak power of nearly $1 \mathrm{MW}$. Absorption of the narrow-band frequency-doubled light pulses leads-to an excitation of the $S_{1}$ electronically excited state of the clusters within the molecular beam. A second broadband laser pulse ionizes the excited clusters. Both light beams interact perpendicularly with the molecular beam so that Doppler broadening is reduced to some 
$40 \mathrm{MHz}$ and sharp intermediate state spectra can be obtained when the first laser is scanned. The ions are mass selected and detected in a linear time of flight mass spectrometer with a resolution of $m / \Delta m \approx 250$.

With this technique we were able to resolve the rotational structure in the vibronic bands of benzene-noble-gas dimers and trimers. This allows us to determine the structure and the exact bond lengths of these complexes.

The high spectral and mass selectivity achievable is demonstrated in Fig. 15.4 by the Doppler-free spectra of the strongest one-photon vibronic band, $6_{0}^{1}$, of two isotopic benzene-noble-gas dimers $\mathrm{C}_{6} \mathrm{H}_{6}{ }^{84} \mathrm{Kr}$ and $\mathrm{C}_{6} \mathrm{H}_{6}{ }^{86} \mathrm{Kr}$ [15.15]. By separate integration of the ion current at the different isotopic masses, both spectra are measured simultaneously within the natural isotopic mixture of $\mathrm{Kr}$. The complexes are produced by expansion of 6-mbar benzene seeded in 5-bar $\mathrm{Kr}$ through a pulsed $300-\mu \mathrm{m}$ nozzle. Since the natural abundance of ${ }^{86} \mathrm{Kr}$ $(17.3 \%)$ is only one third of ${ }^{84} \mathrm{Kr}(57.0 \%)$, the vertical scale of the upper spectrum in Fig. 15.4 is expanded by a factor of 3 .

Both hands display the rotational structure of a prolate symmetric top with a rotational temperature $T_{\text {rot }} \approx 1.5 \mathrm{~K}$. On the low- and high-energy side of the spectrum, well-resolved strong lines appear which are assigned to the $\mathrm{P}$ and $\mathrm{R}$ branch, respectively. The seven strong features in the center of the band are subbranches of the $\mathrm{Q}$ branch with partly overlapping rotational lines. The blueshaded wings of the subbranch originate from lines with constant $K^{\prime}$ and $\Delta K$ but varying $\mathbf{J}^{\prime}$ and indicate an increase of the $B$ rotational constant in the $S_{1}$ state. Due to the small relative mass change between the two clusters of only $1.2 \%$, the differences in band structure and line positions cannot be seen by naked eye, but only by detailed analysis of the data.

For a precise determination of the rotational constants a two-stage computer fit to the line positions was performed, according to a rigid symmetric top energy formula. In the first step, the ground state constant $B_{0}^{\prime \prime}$ is evaluated by combination differences $\left[B_{0}^{\prime \prime}=0.026562(13) \mathrm{cm}^{-1}\right.$ for $\mathrm{C}_{6} \mathrm{H}_{6} \cdot{ }^{84} \mathrm{Kr}$ and $B_{0}^{\prime \prime}$ $=0.026263(21) \mathrm{cm}^{-1}$ for $\mathrm{C}_{6} \mathrm{H}_{6}{ }^{86} \mathrm{Kr}$ ], i.e. frequency differences of transitions

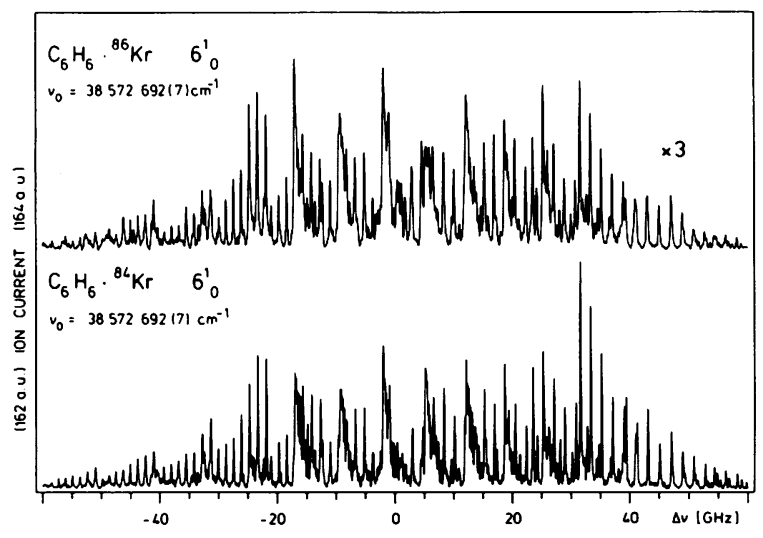

Fig. 15.4. Mass-selected rotationally resolved UV spectrum of the $6_{0}^{1}$ band of the $\mathrm{C}_{6} \mathrm{H}_{6} \cdot{ }^{86} \mathrm{Kr}$ (upper trace) and $\mathrm{C}_{6} \mathrm{H}_{6} \cdot{ }^{84} \mathrm{Kr}$ (lower trace) van der Waals clusters. The two spectra were measured for $\mathrm{Kr}$ in natural isotopic abundance. Note the increased vertical scale of the less abundant $\mathrm{C}_{6} \mathrm{H}_{6}{ }^{.86} \mathrm{Kr}$ isotopic cluster 


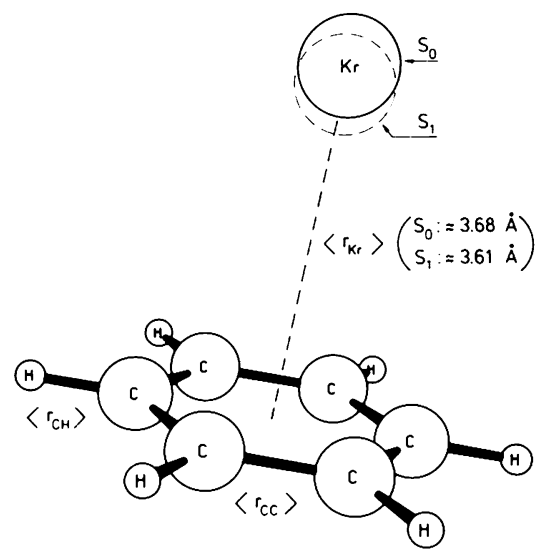

Fig. 15.5 Structure of the $\mathrm{C}_{6} \mathrm{H}_{6}{ }^{86} \mathrm{Kr}$ van der Waals cluster. The $\mathrm{Kr}$ atom is located on the $\mathrm{C}_{6}$ symmetry axis of the benzene molecule. The bond length of $3.68 \AA$ in the electronic ground state decreases by $70 \mathrm{~m} \AA$ upon electronic excitation of benzene

starting at the same ground state but leading to different excited states. In a second step the excited state constants $A_{v}^{\prime}\left[0.090838(5) \mathrm{cm}^{-1}\right], B_{v}^{\prime}$ $\left[0.027266(15) \mathrm{cm}^{-1}\right.$ for $\mathrm{C}_{6} \mathrm{H}_{6} \cdot{ }^{.84} \mathrm{Kr}$ and $0.026962(24) \mathrm{cm}^{-1}$ for $\left.\mathrm{C}_{6} \mathrm{H}_{6}{ }^{\cdot 86} \mathrm{Kr}\right]$, and $\zeta_{\text {eff }}[-0.5860(3)]$ are fitted to about 100 unblended rotational lines of the experimental spectrum. By means of a simultaneously measured iodine absorption spectrum, it is possible to fix the band origin, $v_{0}$, to an accuracy of $0.007 \mathrm{~cm}^{-1}$ (see Fig. 15.4). The standard deviation of the fit is $23 \mathrm{MHz}$, which is less than a fifth of the spectral linewidth.

The low standard deviation and the absence of an asymmetry splitting of the lines with low $K$ values within the experimental resolution demonstrate that the $\mathrm{C}_{6} \mathrm{H}_{6} \cdot \mathrm{Kr}$ cluster is a prolate symmetric top, with the $\mathrm{Kr}$ atom located above the benzene ring on the $C_{6}$ rotational axis (Fig. 15.5). From the vibrationally averaged rotational constants $B_{0}^{\prime \prime}$ and $B_{v}^{\prime}$, accurate values for the effective bond distance $\left\langle r_{\mathrm{Kr}}\right\rangle$ in the $\mathrm{S}_{0}$ and the $\mathrm{S}_{1}$ state are calculated. They are identical for both isotopic species and are given in Fig. 15.5. Due to a slightly larger ring size and a higher polarizability of benzene in the $S_{1}$ state, the bond distance decreases upon electronic excitation.

Recently, similar experiments on benzene- $\mathrm{Ar}_{2}$ have shown that the second Ar atom is located in the symmetrical position on the other side of the benzene ring [15.16]. No change in the benzene-Ar distance is found when the second $\mathrm{Ar}$ atom is adsorbed to the benzene surface. This points to a vanishing three-body interaction between the two Ar atoms in the cluster through the benzene plane. Such data represent basic information necessary for a microscopic understanding of the van der Waals interaction in organic molecules.

\subsection{Summary and Conclusion}

In this note we have shown that Doppler-free spectroscopy is now feasible for large molecular systems. Using narrow-band dye lasers, Doppler-free spectra 
were measured either by two-photon absorption from counterpropagating light beams in a room temperature gas sample or by expansion and cooling of a highpressure gas sample through a nozzle, reducing the Doppler broadening. Analysis of these spectra provides hitherto inaccessible information on molecular structure and intramolecular dynamics. Structural information is particularly interesting for weakly bound molecular clusters. In this way a microscopic understanding of solvation and complexation processes is expected in the near future.

\section{References}

15.1 P.P. Sorokin, J.R. Lankard: IBM J. Res. Develop. 10, 162 (1966)

15.2 F.P. Schäfer, W. Schmidt, J. Volze: Appl. Phys. Lett. 9, 306 (1966)

15.3 V.S. Letokhov, V.P. Chebotayev: Nonlinear Laser Spectroscopy (Springer, Berlin, Heidelberg 1977)

15.4 E. Riedle, H.J. Neusser, E.W. Schlag: J. Chem. Phys. 75, 4231 (1981)

15.5 F. Biraben, M. Bassini, B. Cagnac: J. de Phys. 40, 445 (1979)

15.6 E. Riedle, R. Moder, H.J. Neusser: Opt. Commun. 43, 388 (1982)

15.7 L.S. Vasilenko, V.P. Chebotayev, A.V. Shishaev: JETP Lett. 12, 113 (1970)

15.8 E. Riedle, H.J. Neusser: J. Chem. Phys. 80, 4686 (1984)

15.9 T.W. Hänsch, B. Couillaud: Opt. Commun. 35, 441 (1980)

15.10 J.H. Callomin, T.M. Dunn, I.M. Mills: Phil. Trans. Roy. Soc. London A 259, 499 (1966)

15.11 H. Sieber, E. Riedle, H.J. Neusser: J. Chem. Phys. 89, 4620 (1988)

15.12 G. Herzberg: Molecular Spectra and Molecular Structure, III. Electronic Spectra and Electronic Structure of Polyatomic Molecules (van Nostrand Reinhold, New York 1966)

15.13 D.H. Levy: Ann. Rev. Phys. Chem. 31, 197 (1980)

15.14 Th. Weber, A. von Bargen, E. Riedle, H.J. Neusser: J. Chem. Phys. 92,90 (1990)

15.15 Th. Weber, E. Riedle, H.J. Neusser, E.W. Schlag: Chem. Phys. Lett. (1991) in press

15.16 Th. Weber, H.J. Neusser: J. Chem. Phys. 94, 7689 (1991)

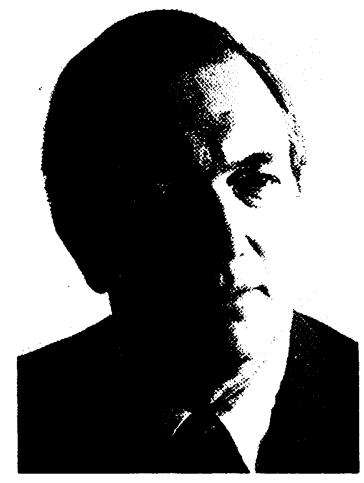

Edward W. Schlag

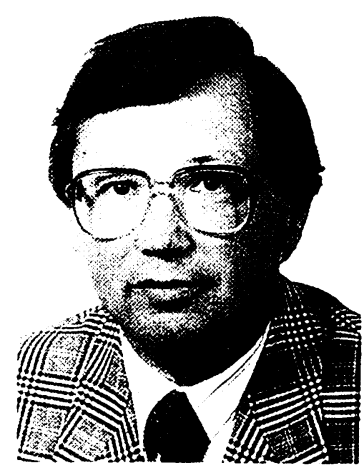

Hans Jürgen Neusser 